Objectives To address the limitations of existing studies, and evaluate the effect of a controlled, long-term (24 week intervention, 24 week follow-up), intensive (1 hour physiotherapy twice weekly, and home-exercise for 1 hour $5 \mathrm{x}$ weekly), tailored physiotherapy program to improve muscle strength, endurance and deep stabiliser system, and quality of life/disability in cohorts with a substantial number of IIM patients.

Methods All patients fulfilled the Bohan and Peter 1975 diagnostic criteria, had muscle involvement, and were consecutively recruited between 2014-2016. At months 0,3,6,12 all patients were assessed by a physician (physical examination, MITAX, MYOACT, and MDI), and a physiotherapist blinded to intervention (MMT-8 - muscle strength test, FI-2 - muscle endurance test), patients filled out patient reported outcomes (PRO)/questionnaires (HAQ, SF-36, Beck's depression inventory-II (BDI-II), PROs assessing nutrition and fatigue), body composition was analysed using densitometry (iDXA Lunar) and bioelectric impedance (BIA2000-M), and patients provided blood for routine laboratory analysis and biobanking. Normality of data was tested and inter-group analysis performed with 2-way ANOVA and intra-group analysis by Friedman's test with Dunn's post hoc test.

Results 27 IIM patients were recruited into the intervention group (IG) and 27 patients into the control group (CG). Compared to observed statistically significant deterioration in CG over the period of months $0-6$, we found statistically significant improvement in FI-2, MMT8, HAQ, BDI-II. Only numerical improvement in IG compared to numerical deterioration in CG, which has not reached statistical significance, was observed in SF-36 and fatigue PROs.

Conclusions Our physiotherapy program led to a significant improvement in muscle strength, endurance, and function. Additionally, patients expressed a decrease in the level of depression. The improvements were clinically meaningful in a substantial proportion of patients.

Acknowledgements Supported by AZV-16-33574A.

Disclosure of interest None declared

\section{P100 THE LINK BETWEEN ANGIOGENESIS AND OSTEOGENESIS IN SPONDYLOARTHRITIS}

\begin{abstract}
1,2 $\mathrm{MH}$ Kaaij ${ }^{*}, 1,2 \mathrm{PP}$ van Hamburg, ${ }^{3,4} \mathrm{G}$ Kollias, ${ }^{1,2} \mathrm{D}$ Baeten, 1,2 $\mathrm{L}$ van Duivenvoorde. ${ }^{1}$ Amsterdam Rheumatology and immunology Centre; ${ }^{2}$ Department of Experimental Immunology, AMC/University of Amsterdam, Amsterdam, Netherlands; ${ }^{3}$ Division of Immunology, Biomedical Sciences Research Centre 'Alexander Fleming', Vari; ${ }^{4}$ Department of Physiology, National and Kapodistrian University of Athens, Athens, Greece
\end{abstract}

\subsection{6/annrheumdis-2018-EWRR2018.116}

Introduction Spondyloarthritis is characterised by inflammation, extensive angiogenesis and pathological osteogenesis. Transmembrane (tm) TNF transgenic (tg) mice ${ }^{1}$ that overexpress tmTNF exhibit features of SpA, including chronic inflammation and pathological osteogenesis. tmTNF ligation to TNF receptor 2 in endothelial cells (ECs) can induce signal transduction pathways, that may promote these processes. Of note, angiogenesis and osteogenesis are coupled by EC differentiation towards a type $\mathrm{H}$ ${\text { (CD } 31^{\text {hi }} \text { endomucin }}^{\text {hi }}$ ) phenotype. ${ }^{2}$

Objectives To investigate the link between pathological angiogenesis and osteogenesis in tmTNF tg mice and the potential contribution of noncanonical NF- $\mathrm{KB}$ signalling in ECs to this process and infiltration of immune cells into the bone marrow (BM).

Methods Vertebrae and ankles from 6 and 12 weeks and 8 months old tmTNF tg mice or sex-matched non-tg littermates $(\mathrm{n}=18)$ were prepared by cutting $60 \mu \mathrm{m}$ thick cryosections for confocal imaging.

Results tmTNF tg mice exhibited ectopic osteogenesis which was not observed in non-tg littermates. Immunostainings showed that type $\mathrm{H}$ vessels are in the vicinity of the ectopic osteogenesis and osterix ${ }^{+}$osteoprogenitors. At six weeks of age, osterix ${ }^{+}$cells are located throughout the ectopic lesion, while at eight months, osterix ${ }^{+}$cells are only present at the border of the lesion. Furthermore, there is increased osteogenesis and a different vessel architecture within the vertebrae of tmTNF tg mice compared to non-tg littermates that progresses with age. Non-tg littermate vertebrae only have physiological osteogenesis, which is in the metaphysis and periosteum. In addition, tmTNF tg mice also exhibit altered bone marrow (BM) architecture containing extensive lymphoid aggregates, which predominantly consisted of $\mathrm{B} 220^{+} \mathrm{B}$ cells.

Conclusions tmTNF overexpression in mice leads to development of type $\mathrm{H}$ vessels associated with ectopic osteogenesis. In addition, extensive lymphoid aggregates develop in the BM. Current studies are aimed at identification of signalling pathways in ECs that contribute to these processes.

\section{REFERENCES}

1. Alexopoulou L, et al. Eur J Immunol 1997;27(10):2588-92.

2. Kusumbe AP, et al. Nature 2014;507(7492):323-328.

\section{Disclosure of interest None declared}

\section{P101 DOT1L INHIBITION INCREASES DERMAL FIBROBLAST PROLIFERATION BUT HAS NO EFFECTS ON IN VITRO OR IN VIVO COLLAGEN DEPOSITION IN MODELS OF FIBROSIS} ${ }^{1,2} \mathrm{~N}$ Berghen* ${ }^{*},{ }^{1,2}$ E De Langhe, ${ }^{1,2} \mathrm{R}$ Lories. ${ }^{1}$ Department of Development and Regeneration,
KU Leuven; ${ }^{2}$ Division of Rheumatology, UZ Leuven, Leuven, Belgium

10.1136/annrheumdis-2018-EWRR2018.117

Introduction Fibrosis is a key process in Systemic Sclerosis, a devastating disease that is not well understood. The role of epigenetic disease mechanisms is increasingly explored. DOT1L, the unique H3K79-methyltransferase, methylates histone 3 at the Lysine residue at position 79, and regulates gene expression. Inhibition of DOT1L has cell-type specific effects on Wnt-signalling, a pathway suggested to play an important role in fibrosis.

Objectives To study the role of DOT1L in fibrosis.

Methods Primary cell cultures of fibroblasts, isolated from healthy human skin and treated with DOT1L-inhibitor EPZ5676 or vehicle for 14 days, were stimulated with TGF $\beta$ $5 \mathrm{ng} / \mathrm{ml}$ after starvation. Smooth muscle alpha 2 actin (ACTA2) gene expression was measured by RT-qPCR. Efficiency of DOT1L-inhibition was analysed with Western Blot for dimethylated H3K79. Picrosirius Red staining measured collagen I and III deposition. Proliferation was analysed with 5-Bromo-2'-deoxy-uridine (BrdU) labelling. 7-9 week old Col1a2; Cre-ERT ${ }^{2}$; DOT1 $11^{\mathrm{fl} / \mathrm{fl}}$ mice were injected intraperitoneally with tamoxifen ( $1 \mathrm{mg} /$ day, 5 days) to induce a fibroblastspecific DOT1L knockout. Bleomycin $(0.1 \mathrm{mg})$ or vehicle was injected subcutaneously (5 days/week, 4 weeks). Injected skin was analysed by $\mathrm{OH}$-prolin assay for collagen content, and by histology for dermal thickness measurement.

Results The DOT1L-inhibitor EPZ-5676 reduced H3K79 dimethylation in all samples. TGF $\beta$ increased expression of ACTA2 
but 3 out of 4 DOT1L inhibited samples showed a significantly reduced effect of TGF $\beta$ on ACTA2 expression. The amount of collagen I and III in the extracellular matrix after 72 hours of TGF $\beta$, was comparable between control and EPZ treated fibroblasts. BrdU labelling assay showed increased proliferation with DOT1L inhibition. In vivo, subcutaneous bleomycin induced an increased dermal thickness and skin collagen content in mice. No difference in the effect of bleomycin was found between mice with a conditional fibroblastspecific DOT1L knockout or wild type mice.

Conclusions In an in vitro model of fibrosis, primary human dermal fibroblasts treated with a DOT1L-inhibitor showed increased proliferation and reduced upregulation of ACTA2 but did not result in detectable differences in collagen deposition. In an in vivo murine model of skin fibrosis, no difference in bleomycin-induced skin thickness and collagen content was found when DOT1L was knocked out in fibroblasts.

Disclosure of interest None declared

\section{P102 S100A9 HAMPERS OSTEOCLAST DIFFERENTIATION FROM CIRCULATING PRECURSORS BY REDUCING THE EXPRESSION OF RANK}

${ }^{1} \mathrm{M}$ van den Bosch, ${ }^{1} \mathrm{I}$ Di Ceglie, ${ }^{2} \mathrm{~T}$ Vogl, ${ }^{2} \mathrm{~J}$ Roth, ${ }^{3} \mathrm{C}$ Goodyear, ${ }^{1} \mathrm{P}$ van der Kraan, ${ }^{1} \mathrm{~A}$ Blom, ${ }^{1} \mathrm{P}$ van Lent* ${ }^{*}{ }^{1}$ Radboud university medical centre, Nijmegen, Netherlands; ${ }^{2}$ University of Muenster, Muenster, Germany; ${ }^{3}$ University of Glasgow, Glasgow, UK

\subsection{6/annrheumdis-2018-EWRR2018.118}

Introduction The alarmins S100A8/A9 are produced in high levels in the synovium during both experimental and human rheumatoid arthritis (RA) and have been implicated in inflammation-induced bone resorption. We and others have previously shown that stimulation of mature osteoclasts with S100A8/A9 results in increased numbers and resorptive activity. In agreement, reduced bone destruction was observed after induction of experimental RA models in S100a $9^{-/-}$mice. However, the effects of S100A8/A9 on monocyte-to-osteoclast differentiation remain elusive.

Objectives Here, we investigated the effects of S100A9 on CD $14^{+}$monocytes and their potential to differentiate into osteoclasts.

Methods CD14 ${ }^{+}$monocytes were isolated from buffy coats of healthy donors using density gradient centrifugation and magnetic cell sorting. Cells were differentiated into osteoclasts with macrophage colony-stimulating factor (M-CSF) and Receptor activator of nuclear factor kappa-B (RANK) ligand (RANKL) in the presence or absence of S100A9. mRNA expression was determined by RT-qPCR and protein expression was determined using Luminex analysis. Moreover, osteoclast differentiation was assessed using Tartrate-resistant acid phosphatase (TRAP) staining and the resorptive capacity was determined using mineral-coated plates. RANK protein expression was assessed using FACS.

Results We observed that S100A9 stimulation of monocytes resulted in a strong induction of various pro-inflammatory factors, such as interleukin (IL)1 $\beta$, IL6, IL8, and tumour necrosis factor (TNF) $\alpha$ after 24 hour, both on the mRNA and protein level. Interestingly, we observed a strong decrease in the number of multinucleated osteoclasts as determined by TRAP staining, at day 6 and 8 after start of the cultures. In agreement with this, the cells showed a strongly reduced resorptive capacity. We demonstrated that already a 24 hour stimulation with S100A9 strongly reduced the osteoclastogenic potential of the $\mathrm{CD}^{+} 4^{+}$monocytes. Finally, we observed that S100A9 stimulation hampered the M-CSF-induced upregulation of RANK, which could be reversed by addition of the TNF $\alpha$ inhibitor etanercept, but not the interleukin 1 receptor antagonist.

Conclusions Whereas S100A8/A9 have been previously shown to stimulate the numbers and resorptive capacity of mature osteoclasts, we here show that stimulation of monocytes with S100A9 strongly inhibits their osteoclastogenic potential, possibly via $T N F \alpha$-induced reduction of RANK expression. This suggests that the timing of exposure to S100A8/A9 is an important determinant for monocyte-to-osteoclast differentiation.

Disclosure of interest None declared

\section{P103 PRO-FIBROTIC RESPONSES INDUCED BY THYMIC STROMAL LYMPHOPOETIN}

${ }^{1} \mathrm{~L}$ Wang, ${ }^{2} \mathrm{~S}$ Wang, ${ }^{3} \mathrm{~V}$ Taneja, ${ }^{2} \mathrm{R}$ Vassallo. 'Department of Pulmonary Medicine, Guang'anmen Hospital, China Academy of Chinese Medicine Science, Beijing, China; ${ }^{2}$ Pulmonary and Critical Care Medicine; ${ }^{3}$ Immunology, Mayo Clinic, Rochester, Minnesota, United States

\subsection{6/annrheumdis-2018-EWRR2018.119}

Registration status I still need to register to EWRR Introduction Thymic Stromal Lymphopoetin (TSLP) is a T helper-2 (Th2) cytokine that may be an important regulator of tissue fibrosis. ${ }^{1}$ Rheumatoid Arthritis (RA)-associated interstitial lung disease is a fibrotic extra-articular complication associated with dysregulated adaptive immunity in the lung. In a recent study - Vassallo et al. Clin Immunol 2014;152(1-2):25-35 we reported increased TSLP mRNA levels in the lungs of cigarette smoke-exposed, collagen induced arthritis mice with a DQ8 background. ${ }^{2}$

Objectives The purpose of this study was to explore direct molecular mechanisms by which TSLP induces fibrogenesis.

Methods We performed in vitro studies with human lung fibroblasts (Lonza) incubated with recombinant human TSLP (R\&D Systems, 0-10 ng/ml) and determined TSLP-induced proliferation using a CyQuant assay, as well as collagen and fibronectin gene expression. We also investigated signaling intermediates activated by TSLP utilizing a Signal Transduction and Activator of Transcription (STAT)1-6 ELISA assay on lysates generated from TSLP stimulated fibroblasts at varying time points.

Results Human lung fibroblasts proliferate directly in response to TSLP with concentrations $\geq 1 \mathrm{ng} / \mathrm{ml}$ inducing a significant proliferative response compared with controls. TSLP also induced fibronectin and collagen gene expression in lung fibroblasts. Incubation with TSLP induced phosphorylation of STAT-1 and STAT-3: phosphorylation of STAT-2, as well as 4-6 was not observed. Phosphorylation of STAT-1 and 3 were observed within 30 minutes of incubation with TSLP (10 ng/ $\mathrm{ml})$.

Conclusions These in vitro studies demonstrate that fibroblasts are directly responsive to recombinant human TSLP which induces proliferation and matrix generation, potentially through activation of STAT-1 and 3. These observations further support a role for TSLP as a regulator of tissue fibrosis in the lung, and suggest a potential therapeutic target for the treatment of autoimmunity related fibrosis. 